\title{
Power quality event classification using complex wavelets phasor models and customized convolution neural network
}

\author{
Likhitha Ramalingappa, Aswathnarayan Manjunatha \\ Department of Electrical and Electronics Engineering, Sri Krishna Institute of Technology, Bengaluru, India
}

\section{Article Info}

Article history:

Received Jan 25, 2021

Revised Jul 29, 2021

Accepted Aug 9, 2021

\section{Keywords:}

Complex wavelets

Deep learning

PQ events classification

Smart grid

Voltage dips

ABSTRACT

Origin and triggers of power quality (PQ) events must be identified in prior, in order to take preventive steps to enhance power quality. However it is important to identify, localize and classify the PQ events to determine the causes and origins of PQ disturbances. In this paper a novel algorithm is presented to classify voltage variations into six different PQ events considering the space phasor model (SPM) diagrams, dual tree complex wavelet transforms (DTCWT) sub bands and the convolution neural network (CNN) model. The input voltage data is converted into SPM data, the SPM data is transformed using 2D DTCWT into low pass and high pass sub bands which are simultaneously processed by the $2 \mathrm{D}$ CNN model to perform classification of PQ events. In the proposed method CNN model based on Google Net is trained to perform classification of PQ events with default configuration as in deep neural network designer in MATLAB environment. The proposed algorithm achieve higher accuracy with reduced training time in classification of events than compared with reported PQ event classification methods.
\end{abstract}

This is an open access article under the $\underline{C C B Y-S A}$ license.

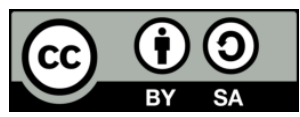

\section{Corresponding Author:}

Likhitha Ramalingappa

Department of Electrical and Electronics Engineering, Sri Krishna Institute of Technology

Chimney Hills No. 29, Hesaraghatta Main Road, Chikkabanavara, Bengaluru, 560090, India

Email: likhitha.pes@gmail.com

\section{INTRODUCTION}

The present power grid is being updated to the so-called smart grid (SG) with the use of information and communication technology, enhanced control systems, and sensing methods [1]. With smart grid technology, there will be 3-40\% of renewable energy resources (RES) integrated by 2030. The evolution of the smart grid mechanism gives rise to new challenges in power quality issues [2], [3]. Sources of disturbances both at the source end and the consumer side needs to be found and suitably addressed to take appropriate mitigation action [4]. IEC 61000-4-30 specifies a power quality (PQ) event and suggests intelligent monitoring of PQ events in order to respond to electrical equipment failures [5].

An appreciable amount of effort is recorded in the literature for analyzing PQ event classification. Classification of PQ events entails the use of feature extraction methods like "Fourier transforms (FT), S transforms (ST), Hilbert Huang transforms (HHT), and Wavelet transforms (WT)" based methods are reported in [6]-[9]. Power quality disturbance (PQD) classification is also heavily influenced by artificial intelligence techniques such as "support vector machine (SVM), artificial neural networks (ANN), fuzzy logic (FL), genetic algorithm (GA), deep learning" based methods are reported in [10]-[12]. Classification of harmonics is carried out by k-means algorithms and support vector machines (SVM) in [13]. Voltage dips and interruptions are classified using expert systems in [14], [15]. Discrete wavelet transforms (DWT) combined with SVM for the categorization of PQ events is described in [16] uses image files of PQ events 
instead of sampled voltage data. Deep learning algorithms are used to classify image data demonstrating $100 \%$ accuracy. Balouji and Salor [17] phase space reconstruction (PSR) and convolutional neural networks $(\mathrm{CNN})$ are used in a hybrid technique for PQ event classification that processes 2D image files representing voltage disturbances demonstrating more accurate results compared with existing methods. A complete framework for PQ event classification using 2D CNNs and space phasor model (SPM) is presented in [18]. In the proposed approach in order to reduce the complexity and improve efficiency in PQ event classification the input voltage data is converted into SPM data, the SPM data is transformed using 2D DTCWT into low pass and high pass sub bands. The low pass sub band and combined high pass sub bands are simultaneously processed by the 2D CNN model to perform classification of PQ events.

\section{RESEARCH METHOD}

\subsection{Space phasor model}

SPM denoted as St, considering the voltages from three phases to neutral $\mathrm{Va}(\mathrm{t}), \mathrm{Vb}(\mathrm{t}), \mathrm{Vc}(\mathrm{t})$ is given as shown in (1) with the parameter $\alpha=\mathrm{e}^{\mathrm{j} 2 \pi / 3}$.

$$
\mathrm{St}=2 / 3\left[\mathrm{Va}(\mathrm{t})+\alpha \mathrm{Vb}(\mathrm{t})+\alpha^{2} \mathrm{Vc}(\mathrm{t})\right], \mathrm{t}=1,2,3 \ldots \ldots . \mathrm{n}
$$

St is a complex quantity representing voltage in time and consists of real and imaginary parameters ranging from -1.1 to $1.1 \mathrm{pu}$. Expression (1) can be rewritten in vector form as $\mathrm{St}=[\mathrm{s} 1, \mathrm{~s} 2, \mathrm{~s} 3 \ldots \mathrm{sn}]^{\mathrm{T}}$. Splitting the vector in terms of real and imaginary vectors and is represented as $S=\left[S_{t, R} S_{t, I}\right]_{n \times 2}$. The parameters $\mathrm{S}_{\mathrm{t}, \mathrm{R}}=\left[\mathrm{S}_{1, \mathrm{R}} \mathrm{S}_{2, \mathrm{R}} \ldots \mathrm{S}_{\mathrm{n}, \mathrm{R}}\right]^{\mathrm{T}}$ and $\mathrm{S}_{\mathrm{t}, \mathrm{I}}=\left[\mathrm{S}_{1, \mathrm{I}} \mathrm{S}_{2, \mathrm{I}} \ldots \mathrm{S}_{\mathrm{n}, \mathrm{I}}\right]^{\mathrm{T}} \varepsilon[-1.1,1.1]$.

SPM plots are represented as identical cycles of voltage waveforms shown in single circle or ellipse and deviations from the normal will have variations in the SPM plots. Classification of voltage dip considering SPM is done by measuring three parameters such as semi-minor, major axis and the major axis direction [19]. These three metrics are linked to voltage dip characteristics considering rotating angle of the ellipse that can be distinguished between 6 types ( $\mathrm{Da}, \mathrm{Db}, \mathrm{Dc}, \mathrm{Ca}, \mathrm{Cb}$ and $\mathrm{Cc}$ ) of voltage dips [20]. The direction of ellipses is changed by $30^{\circ}$ for all the six types of dips generated synthetically. Type $\mathrm{C}$ semicircles $(\mathrm{Ca}, \mathrm{Cb}, \mathrm{Cc})$ denotes phasor with minimum voltage drop and Type $\mathrm{D}$ semicircles (Da, Db, Dc) denotes phasor with major voltage drop. Table 1 presents the labelling of PQ events considering the rotation in the ellipse with rotating angle variation in $30^{\circ}$. The corresponding dip class is also indicated considering the correlation between the rotation angles $\Psi$ and parameter T.

Table 1. PQ event data labelling

\begin{tabular}{cll}
\hline Angles $(\Psi)$ in degrees & T & Class \\
\hline 0 to 30 & 1 & $\mathrm{Db}$ \\
30 to 60 & 2 & $\mathrm{Cc}$ \\
60 to 90 & 3 & $\mathrm{Da}$ \\
90 to 120 & 4 & $\mathrm{Cb}$ \\
120 to 150 & 5 & $\mathrm{Dc}$ \\
150 to 180 & 6 & $\mathrm{Ca}$ \\
\hline
\end{tabular}

Figure 1 presents the example of SPM plots with voltage dip variations, which is represented as ellipses rotated during PQ events and circles during normal events and the dots between the circle and ellipses representing transition segments shown in Figure 1(a). Multi-stage voltage dip SPM diagram is presented in Figure 1(b). If the radius of the circle is less than 1 pu this is considered as last stage event. The two ellipses are defined as Type $\mathrm{C}$ and Type $\mathrm{D}$ voltage dip and the dots correspond to transition segment. Figure 1(c) represents single phase transition of star connected transformer.

The PQ event is represented as ellipse with zero minor axes and also indicates the severity of the event. Figure 1(d) shows the SPM diagram for a type Cc event with high voltage dips in phases a and $b$ and no voltage dips in phase c. Figure 1(e) presents the voltage dip due to transformer energizing process leading to even-harmonic distortion. Hexagon shape of the SPM plot indicates harmonics 5 and harmonics 7 kind of PQ event. Figure 1(f) presents the SPM of PQ event during transients that are indicated as dots oscillating around the circle of normal voltage. Considering the variations in SPM diagrams it is required to suitably classify the PQ events considering the inputs as image sequences. Use of deep learning networks for classification of image sequences is attempted in this work based on SPM diagrams. 


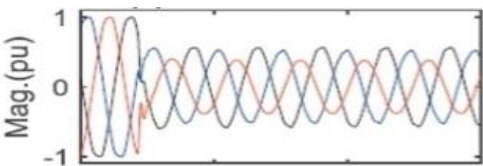

(a)

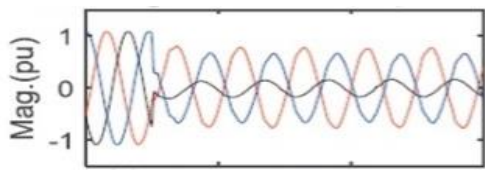

(c)

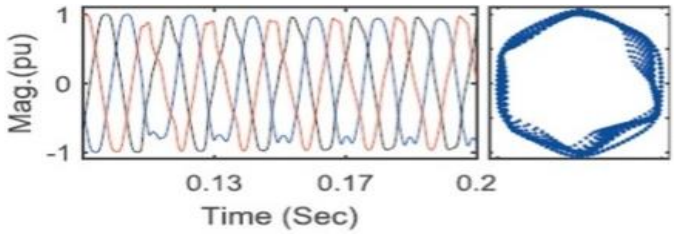

(e)

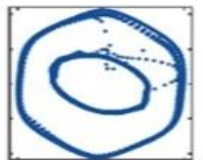

.

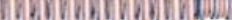
HWWWWWWW

(b)
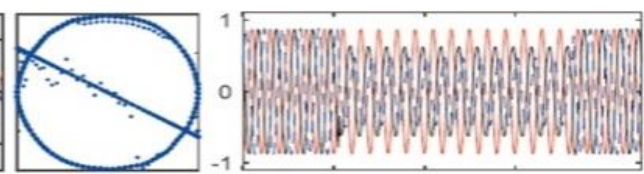

(d)
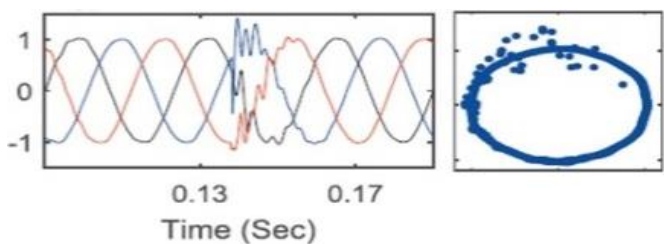

(f)

Figure 1. Waveforms of SPM plots with different voltage dip: (a) voltage dip, (b) multi-stage dip, (c) single-phase interruption, (d) phase-angle jump, (e) harmonic voltage, (f) voltage transient

\subsection{D representation of SPM}

For classification of voltage dips considering SPM diagrams the S vector are quantized into 22 equal length segments defined with matrix $M$ as in (2), where $k_{1}$ and $k_{2}$ are defined as in (3) by using ceiling function ([.]) and absolute value (|.|). Matrix $\mathrm{M}$ is of size $22 \times 22$ that is considered as inputs to the classifier unit.

$$
\begin{aligned}
& M\left(k_{1}, k_{2}\right)=\sum_{i=1}^{n} \quad \sum_{\left(k_{1}, k_{1}\right)} \quad \sqrt{\left(S_{i, R}\right)^{2}+\left(S_{i, I}\right)^{2}} \\
& \left(k_{1}, k_{2}\right)=\left\{\begin{array}{ccc}
\left(\left[10\left(1.1-S_{i, I}\right)\right],\right. & {\left[10\left(1.1-\left|S_{i, R}\right|\right)\right]} & \text { if } S_{i, R}<0 \dot{\&} S_{i, I}>0 \\
\left(\left[10\left(1.1-S_{i, I}\right)\right],\right. & \left.\left[10\left|S_{i, R}\right|\right]\right) & \text { if } S_{i, R}>0 \dot{\&} S_{i, I}>0 \\
\left(\left[10 \mid S_{i, I}\right)\right]+11, & {\left[10\left(1.1-\left|S_{i, R}\right|\right)\right]} & \text { if } S_{i, R}<0 \dot{\&} S_{i, I}<0 \\
\left(\left[10\left|S_{i, I}\right|\right]+11,\right. & \left.\left[10\left|S_{i, R}\right|\right]+11\right) & \text { if } S_{i, R}>0 \dot{\&} S_{i, I}<0
\end{array}\right.
\end{aligned}
$$

In this work, pre-processing is carried out on the SPM data using 2D DTCWT level-1 decomposition obtaining sub bands of complex wavelet. The complex sub bands are processed by the 2D CNN for feature extraction and classification.

\section{CNN AND DUAL TREE COMPLEX WAVELET TRANSFORMS}

The deep learning model-based classification algorithm is designed to process the complex sub bands of input data captured using dual tree complex wavelet transforms.

\subsection{DTCWT algorithm}

The dual tree complex wavelet sub bands are represented by $\psi(\mathrm{x}, \mathrm{y})=\psi(\mathrm{x}) \psi(\mathrm{y})$, where $\psi($.$) is a$ complex wavelet given by $\psi()=.\psi_{\mathrm{h}}()+.\mathrm{j} \psi_{\mathrm{g}}($.$) . The performance of DTCWT, replacing the complex wavelet, is$ articulated as:

$$
\begin{aligned}
\psi(\mathrm{x}, \mathrm{y}) & =\left[\psi_{\mathrm{h}}(\mathrm{x})+\mathrm{j} \psi_{\mathrm{g}}(\mathrm{x})\right]\left[\psi_{\mathrm{h}}(\mathrm{y})+\mathrm{j} \psi_{\mathrm{g}}(\mathrm{y})\right] \\
& =\psi_{\mathrm{h}}(\mathrm{x}) \psi_{\mathrm{h}}(\mathrm{y})-\psi_{\mathrm{g}}(\mathrm{x}) \psi_{\mathrm{g}}(\mathrm{y})+\mathrm{j}\left[\psi_{\mathrm{g}}(\mathrm{x}) \psi_{\mathrm{h}}(\mathrm{y})+\psi_{\mathrm{h}}(\mathrm{x}) \psi_{\mathrm{g}}(\mathrm{y})\right]
\end{aligned}
$$

A set of six complex DTCWT wavelets separated in [21] is given in (5), the real wavelets are represented by $(5 a)$ and $(5 b)$ where $(5 c)$ and $(5 d)$ represents the complex wavelets. In consideration of (5) Table 2 . Offers the 2-D wavelet bases [22] for $i=1,2,3$.

$$
\psi_{\mathrm{i}}(\mathrm{x}, \mathrm{y})=1 \sqrt{ } 2(\psi 1, \mathrm{i}(\mathrm{x}, \mathrm{y})-\psi 2, \mathrm{i}(\mathrm{x}, \mathrm{y}))
$$




$$
\begin{aligned}
& \psi_{\mathrm{i}+3}(\mathrm{x}, \mathrm{y})=1 \sqrt{ } 2(\psi 1, \mathrm{i}(\mathrm{x}, \mathrm{y})+\psi 2, \mathrm{i}(\mathrm{x}, \mathrm{y})) \\
& \psi_{\mathrm{i}}(\mathrm{x}, \mathrm{y})=1 \sqrt{ } 2(\psi 3, \mathrm{i}(\mathrm{x}, \mathrm{y})+\psi 4, \mathrm{i}(\mathrm{x}, \mathrm{y})) \\
& \psi_{\mathrm{i}+3}(\mathrm{x}, \mathrm{y})=1 \sqrt{ } 2(\psi 3, \mathrm{i}(\mathrm{x}, \mathrm{y})-\psi 4, \mathrm{i}(\mathrm{x}, \mathrm{y}))
\end{aligned}
$$

The set of six wavelets described above is useful for isolating six orientations in the input image in a specified direction, and they are contained in the twelve sub bands without artifacts. The SPM image is processed using 2D DTCWT to obtain the sixteen sub bands of which four of them are low pass real sub bands and 12 of them are high pass real sub bands with six directional orientations both in positive and negative directions. Figure 2 presents the DTCWT sub bands of one PQ event of voltage dips. Figure 3 presents the DTCWT sub bands for PQ event with multi-stage voltage dips. The advantages of working with DTCWT sub bands is that the directional orientations in $\pm 45^{\circ}, \pm 15^{0}$ and $\pm 75^{\circ}$ are localized in three different high pass sub bands and it is easier for the CNN model to classify the features. The low pass sub bands confine the input data information with half the resolution of original image and processing the low pass sub bands reduces complexity by $50 \%$.

Table 2. DTCWT sub bands

\begin{tabular}{llll}
\hline \multicolumn{3}{c}{ High pass sub bands } \\
\hline $\mathrm{i}=1$ & Real & $\psi_{1,1}(\mathrm{x}, \mathrm{y})=\varphi_{\mathrm{h}}(\mathrm{x}) \psi_{\mathrm{h}}(\mathrm{y})$ & $\psi_{2,1}(\mathrm{x}, \mathrm{y})=\varphi_{\mathrm{g}}(\mathrm{x}) \psi_{\mathrm{g}}(\mathrm{y})$ \\
& Imaginary & $\psi_{3,1}(\mathrm{x}, \mathrm{y})=\varphi_{\mathrm{g}}(\mathrm{x}) \psi_{\mathrm{h}}(\mathrm{y})$ & $\psi_{4,1}(\mathrm{x}, \mathrm{y})=\varphi_{\mathrm{h}}(\mathrm{x}) \psi_{\mathrm{g}}(\mathrm{y})$ \\
$\mathrm{i}=2$ & Real & $\psi_{1,2}(\mathrm{x}, \mathrm{y})=\psi_{\mathrm{h}}(\mathrm{x}) \varphi_{\mathrm{h}}(\mathrm{y})$ & $\psi_{2,2}(\mathrm{x}, \mathrm{y})=\psi_{\mathrm{g}}(\mathrm{x}) \varphi_{\mathrm{g}}(\mathrm{y})$ \\
& Imaginary & $\psi_{3,2}(\mathrm{x}, \mathrm{y})=\psi_{\mathrm{g}}(\mathrm{x}) \varphi_{\mathrm{h}}(\mathrm{y})$ & $\psi_{4,2}(\mathrm{x}, \mathrm{y})=\psi_{\mathrm{h}}(\mathrm{x}) \varphi_{\mathrm{g}}(\mathrm{y})$ \\
$\mathrm{i}=3$ & Real & $\psi_{1,3}(\mathrm{x}, \mathrm{y})=\psi_{\mathrm{h}}(\mathrm{x}) \psi_{\mathrm{h}}(\mathrm{y})$ & $\psi_{2,3}(\mathrm{x}, \mathrm{y})=\psi_{\mathrm{g}}(\mathrm{x}) \psi_{\mathrm{g}}(\mathrm{y})$ \\
& Imaginary & $\psi_{3,3}(\mathrm{x}, \mathrm{y})=\psi_{\mathrm{g}}(\mathrm{x}) \psi_{\mathrm{h}}(\mathrm{y})$ & $\psi_{4,3}(\mathrm{x}, \mathrm{y})=\psi_{\mathrm{h}}(\mathrm{x}) \psi_{\mathrm{g}}(\mathrm{y})$ \\
\hline
\end{tabular}
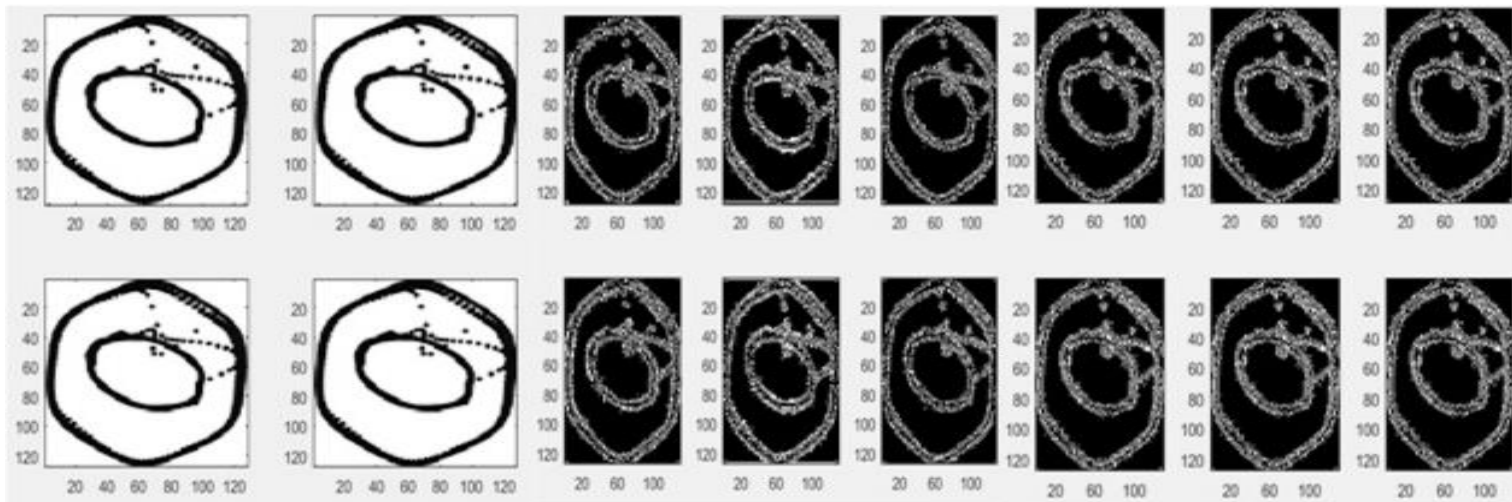

Figure 2. DTCWT sub bands for PQ event with single voltage dip
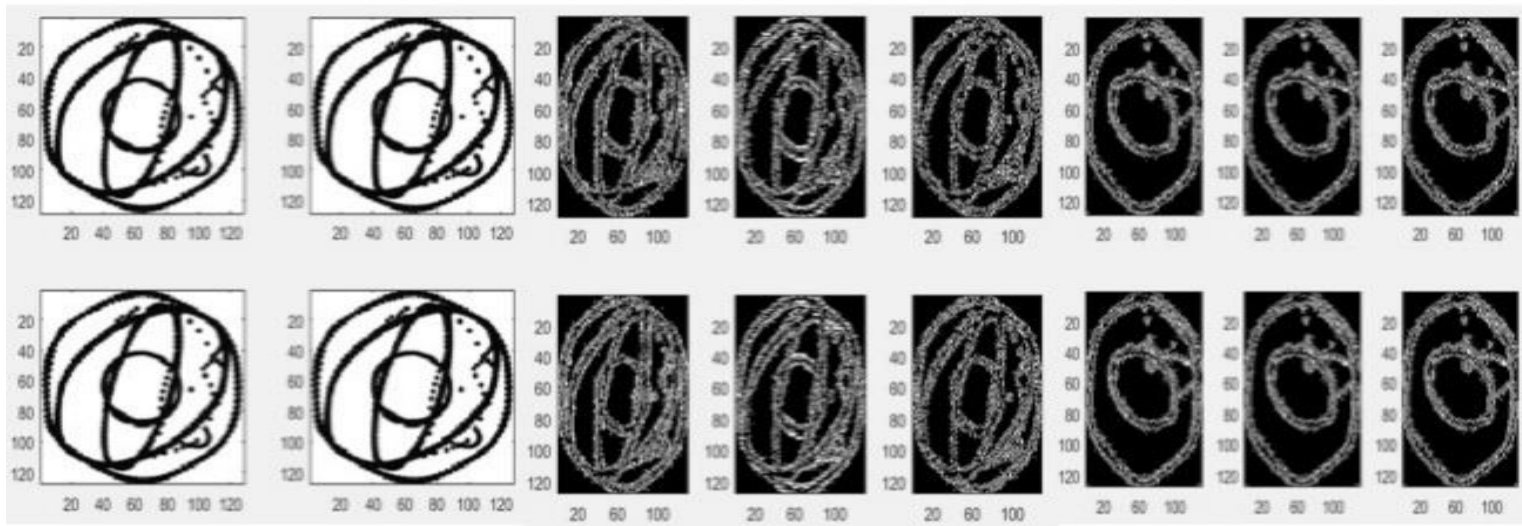

Figure 3. DTCWT sub bands for PQ event with multi-stage voltage dip 


\subsection{CNN model}

The CNN model is derived from extending the feed forward neural network model that comprises of neurons and activation functions in multiple layers. The input layer, pooling layer, convolution layer, fully connected layer and the output layer are included in the CNN model. The convolution layer is a signal processing model that captures low level features in the input data and translates into high level or global features.The signal processing operation in convolution layer is a filtering process that generates feature maps $\mathrm{h}^{\mathrm{k}}$ considering the inputs $\mathrm{x}$ by simple mathematical operation given as in (6). The parameter $\mathrm{W}^{\mathrm{k}}$ and $\mathrm{b}^{\mathrm{k}}$ are the weights and biases of the convolution layer and $\mathrm{f}($.) represents the network activation function.

$$
h^{k} \mathrm{k}_{j}=f\left(W^{k *} x\right)_{i j}+b^{k}
$$

Features extracted from input after every convolution layer are downsized to remove redundancy using the pooling layer using the max-pooling operation. The fully connected layer combines these features to perform classification of the input by considering maximum or average features. The fully connected layer output is given as input to output layer for final classification based on the combined feature maps.

\section{PROPOSED CLASSIFIER BASED ON SPM, DTCWT AND CUSTOMIZED CNN MODEL}

The proposed classifier model is presented in Figure 4. One of the challenges in CNN based classifier is the complexity in data processing as there are several stages of convolution layers, pooling layers and fully connected layers. In order to reduce the complexity and to improve efficiency in PQ event classification the input voltage data is converted into SPM data, the SPM data is converted into low-pass and high-pass sub bands using 2D DTCWT. The low pass and combined high pass sub bands are simultaneously processed by $2 \mathrm{D} \mathrm{CNN}$ model to carry out PQ events classification.

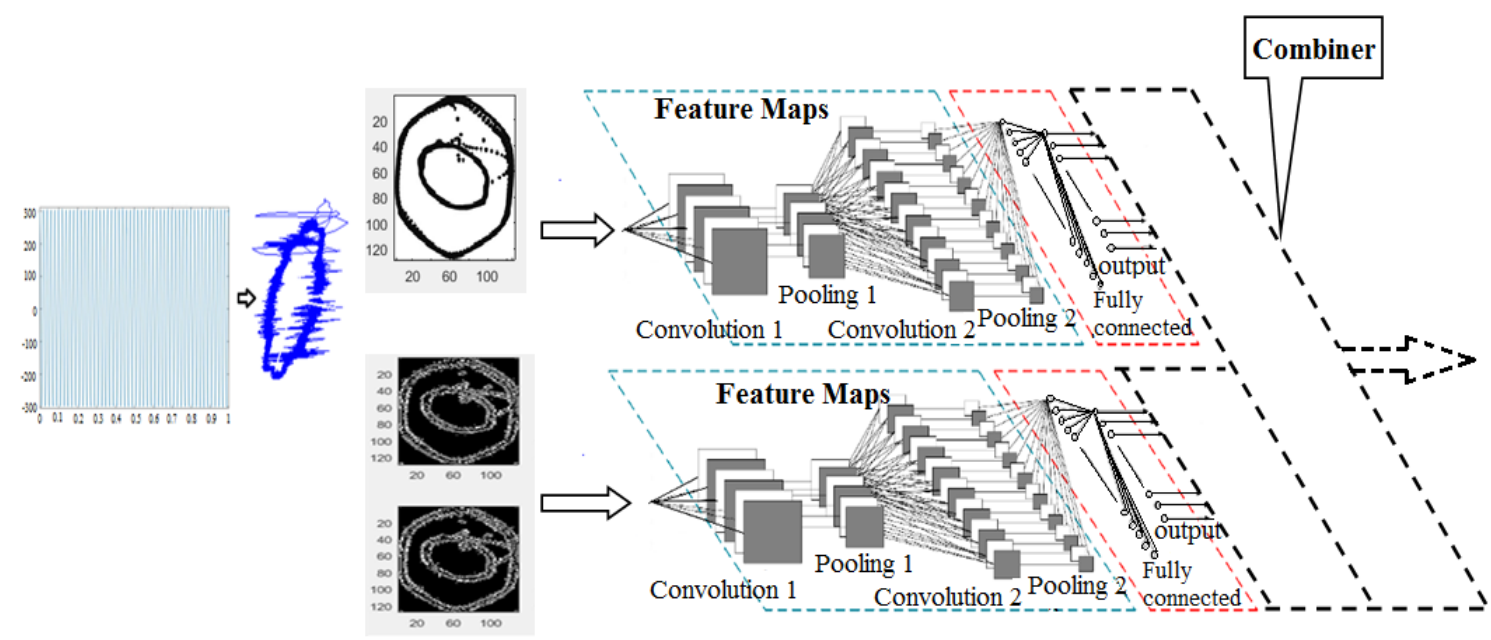

Figure 4. Proposed classifier model combining DTCWT and CNN

The PQ data sets are downloaded from IEEE data port website from which the smart grid data sets from power network of the University of Cadiz that is acquired according to UNE-IEC 61000-4-11:2005 standards using power quality instruments are used for analysis [23]. The CNN model is trained to categorize the input data into any of six classes ( $\mathrm{Da}, \mathrm{Db}, \mathrm{Dc}, \mathrm{Ca}, \mathrm{Cb}$ and $\mathrm{Cc}$ ). The suggested model is evaluated by taking into account two parameters: accuracy and loss, as described in (7).

$$
\begin{aligned}
& \text { Accuracy }=\frac{\text { Correctly predicted dips in all classes }}{\text { Total number of dips }} \\
& \text { Loss }=-\sum_{x} p(x) \ln q(x)
\end{aligned}
$$

The performance assessment of CNN model in performing classification is presented in terms of classification rate (CR) and false alarm rate (FAR) as defined in (8). The parameters TP and TN are true 
positive and true negative for the corresponding data set represented by $\mathrm{i}=1,2,3,4,5,6$. FP and FN are false positive and false negative parameters.

$$
\mathrm{CR}_{\mathrm{i}}=\frac{\mathrm{TP}_{\mathrm{i}}}{\mathrm{TP}_{\mathrm{i}}+\mathrm{FN}_{\mathrm{i}}} \quad \text { and } \quad \mathrm{FAR}_{\mathrm{i}}=\frac{\mathrm{FP}_{\mathrm{i}}}{\mathrm{FP}_{\mathrm{i}}+\mathrm{TN}_{\mathrm{i}}}
$$

In this work CNN model based on Google Net is trained to perform classification of PQ events with default configuration as in deep neural network designer in MATLAB environment and the performances are compared with proposed CNN model. The properties of deep neural network based on GoogLeNet model is presented in Table 3. The GoogLeNet is a 144 layer structure with ReLu network activation function. The flow chart of the CNN model of the most essential layers of GoogLeNet, the input stage, intermediate stage, and output stage, is shown in Figure 5.

Table 3. Properties of GoogLeNet for PQ event classification

\begin{tabular}{ccc}
\hline Layers & Filter Size & Network size \\
\hline Conv+Relu+Pool+Pooldout & $7 \times 7,3 \times 3$ & $224 \times 224 \times 3 \rightarrow 112 \times 112 \times 64$ \\
Conv+Relu & $3 \times 3,3 \times 3$ & $112 \times 112 \times 64 \rightarrow 56 \times 56 \times 64$ \\
Conv+Relu+Cross Conv+Pool & $3 \times 3,3 \times 3,3 \times 3$ & $56 \times 56 \times 64 \rightarrow 28 \times 28 \times 96$ \\
4 stages of parallel layers & 9 stages & $28 \times 28 \times 96 \rightarrow 14 \times 14 \times 24$ \\
Pool+Full Connected+Softmax+Output & $7 \times 7$ & $14 \times 14 \times 24 \rightarrow 7 \times 7 \times 1024$ \\
\hline
\end{tabular}

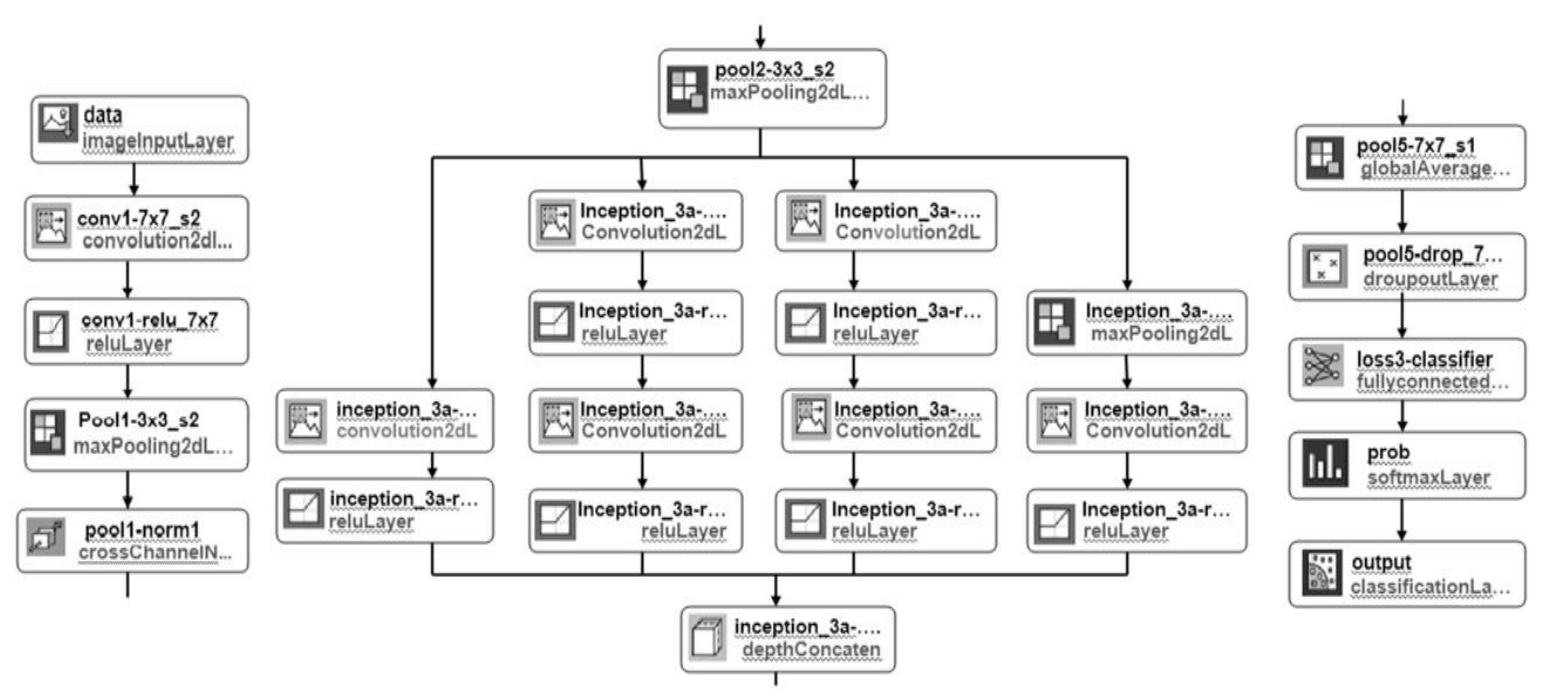

Figure 5. CNN structure: (a) input stage, (b) intermediate stage, (c) output stage

\subsection{DTCWT sub bands and CNN model for classification}

The block diagram of proposed CNN model is presented in Figure 6 that process DTCWT sub bands. The input SPM diagrams are grouped into six different folders in MATLAB environment and each of the diagrams are labelled to distinguish between PQ events. The input data is decomposed into level-1 DTCWT sub bands using 2D DTCWT that uses 10-tap filters. The decomposition generates 6 complex high pass sub bands that are converted to 6 real sub bands by taking absolute values. From 2 complex low pass sub bands one low pass sub band is computed. In order to reduce the complexity in training process the six high pass sub bands are combined into a single image of size $256 \times 384$ by cascading the sub bands (i.e. $128 \times 128$ sized sub bands are arranged into 2 row and 3 columns). The customized CNN model is designed process the low pass sub band (128x128) and high pass sub band (256x384) and the target set is assigned for training the network to perform accurate classification. Each of the SPMs that are in six different folders are processed by the DTCWT module and is used as training data for the customized CNN model. The trained CNN model generate two classification outputs one based on low pass sub bands and the other based on high pass sub bands. Considering two classifier outputs the final decision is carried out to find out the PQ event. The final decision model at the output of CNN is designed to classify the PQ event correctly only if both the CNN outputs classify the PQ event correctly else the classification is termed false. From the training results 
the optimum CNN parameters such as weights and biases are identified and the trained CNN model is evaluated for its performances considering new set of PQ events that are not part of training process.

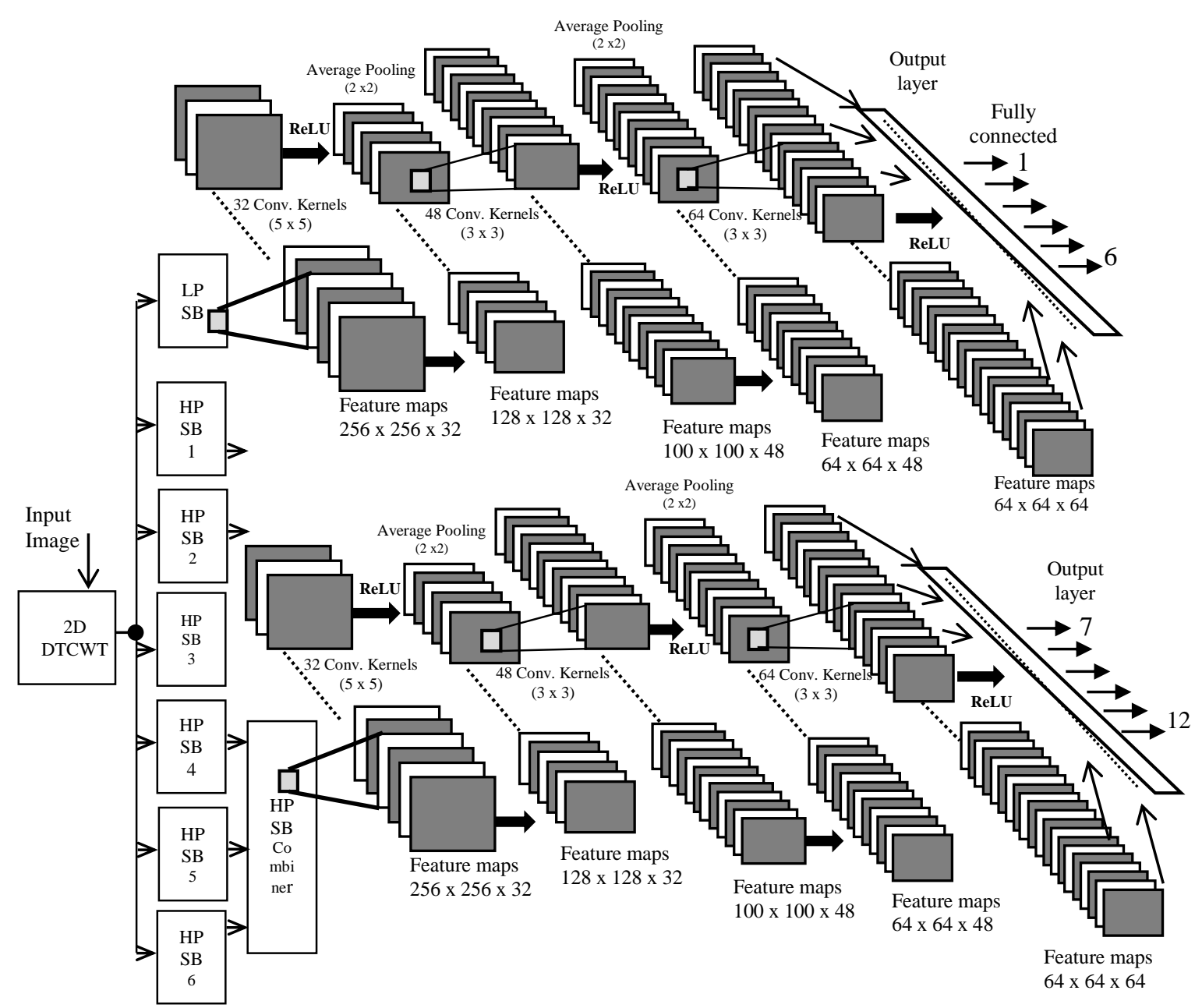

Figure 6. Proposed classifier based on SPM, DTCWT and customized CNN mode

The deep learning networks with the properties are defined in Table 4, configured to train the low pass and high pass sub bands independently. Training the CNN model is carried out by initializing the kernels with default numbers as set by deep network designer toolbox in MATLAB 2020b. The input PQ data obtained from IEEE data port of 480 signals recorded for 3 seconds is split into six different events each of 80 in number. Each of them is stored in different folders with labelling. From 80 data sets $70 \%$ of them are used for training and are labelled and $30 \%$ of them are used for performance evaluation are unlabelled. For each of the data the output and the class to which it belong to known and it is required to estimate the classification correctness after training. The learning algorithm used for calculating the labels specified in this work is the Soft-max activation function. The cost function for calculating the error at the output layer is cross entropy, defined as in (9) that considers the target probability $y_{i}$ and the output probability $\hat{y}_{i}$ in computing the error $e_{i}$.

$$
e_{i}=-\sum_{i=1}^{n} \hat{y}_{i} \ln y_{i}
$$

The error gradients are computed and the weights are updated in the model using back propagation algorithm. The error is minimized and the CNN model parameters such as weights and biases are updated considering gradient descent method. These steps are repeated until all the training images are processed and for the given epoch set during training. The CNN model trained is saved and the network is exported into workspace for testing the network performance. 
Table 4. Properties of GoogLeNet for PQ event classification

\begin{tabular}{ccc}
\hline Layers & Filter Size & Network size \\
\hline Input image & - & $256 \times 262$ \\
2D DTCWT LPSB-4, HPSB-12 & 10 -tap & $128 \times 128$ \\
Sub band selection & - & $128 \times 128,256 \times 384($ LPSB-1, HPSB-1) \\
Conv+Relu+Pool+Pooldout & 32 kernels of 5x5, & $256 \times 256 \times 32 \rightarrow$ \\
& $2 \times 2$ avg. pooling & $128 \times 128 \times 32 \rightarrow 100 \times 100 \times 48$ \\
Conv+Relu+Pool+Pooldout & 48 kernels of 3x3, & $100 \times 100 \times 48 \rightarrow 64 \times 64 \times 48$ \\
Conv + Relu & $2 \times 2$ avg. pooling & $64 \times 64 \times 64$ \\
Full connected+Softmax+Output & 64 kernels of 3x3 & 6,6 LPSB, HPSB \\
Combiner/selector & $6 \times 6$ & 6 classes
\end{tabular}

\section{RESULTS AND DISCUSSION}

The performance of the presented model is evaluated by considering the test data along with synthetically generated another set of PQ events. Figure 7 presents the training performance results in terms of accuracy and loss. More than $90 \%$ of accuracy in classification is achieved only after 10 epochs and the loss factor is almost zero after 10 epochs.

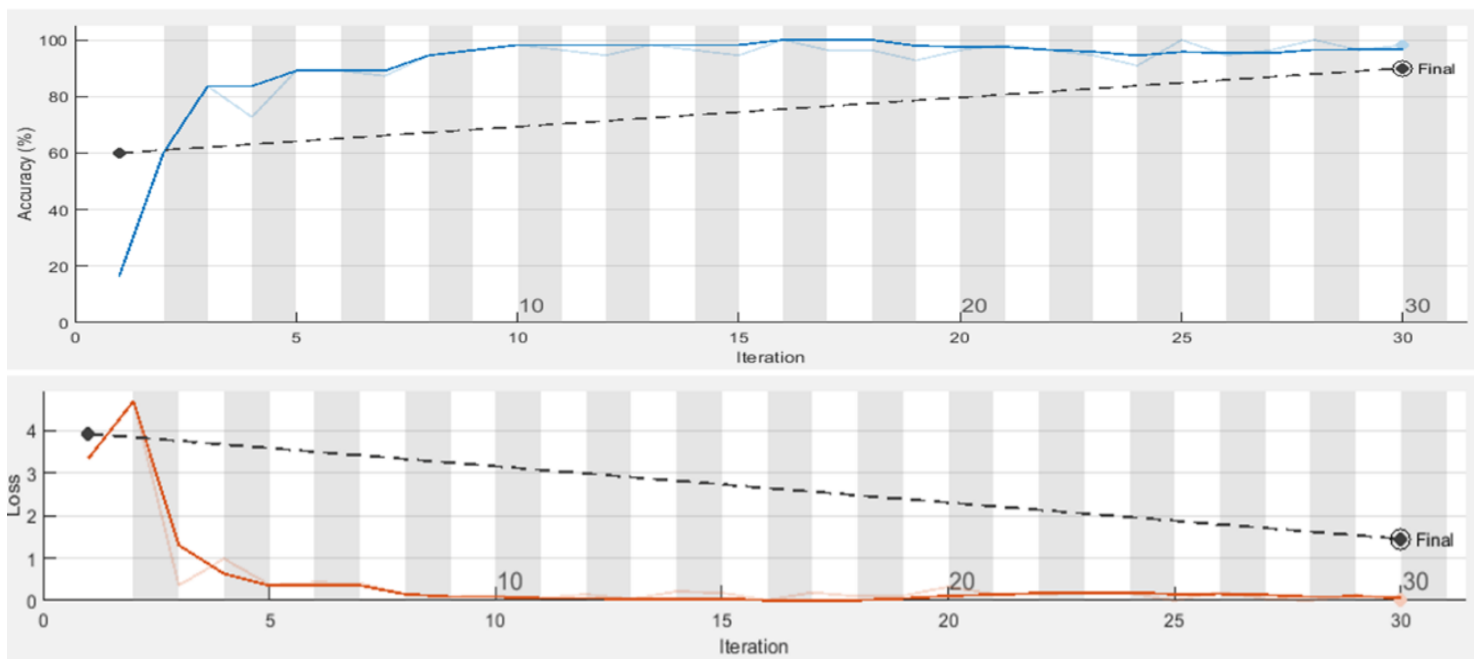

Figure 7. Training performance; (a) accuracy, (b) loss

Validation accuracy of $90 \%$ is achieved in total time of $13 \mathrm{~min} 12 \mathrm{sec}, 30$ epochs and learning rate of 0.01 was set during training process. The total training time for the proposed network is compared with the training time of GoogLeNet results. Intel Core i3-3110M CPU@2.4GHz with 4.0 GB and 64-bit OS is used as the system in which MATLAB2020b is used for modelling and evaluation of the CNN model. Figure 8 depicts the suggested CNN model's confusion matrix. Where the classification accuracy from the test vectors is found to be $99.16 \%$. Figure 9 shows confusion matrix for new data set, it is found that for the data Db and $\mathrm{Ca}$ the CNN model classifies accurately. In case of test data belonging to Cc, 98 data sets are classified correctly, $2 \mathrm{Cc}$ data is classified as Ca. Similarly in case of Dc data of the 100 data sets 92 of them are correctly classified and 8 of them are classified as $\mathrm{Ca}, \mathrm{Cb}$ and $\mathrm{Da}$.

Table 5 presents the performance in terms of CR and FAR. The average CR and FAR is $97.23 \%$ and $0.48 \%$ respectively. Table 6 compares the accuracy, number of PQ events classified, total time for training, and total time for data processing of the proposed CNN model to all other existing work. From the comparison results the advantages of the proposed method is the computation time which is 23 seconds or 94.5\% improvement is achieved. The accuracy of the proposed model in classification is $97.23 \%$ as compared with similar method.

Two factors that give false classification results are the noise in the input data and the size of major axis and minor axis of the ellipse if it is below a certain limits the network is not able to classify appropriately. The classification accuracy can be further improved by further training the network with real time signals considered in large number and setting the learning rare less than 0.001 . The network can be further modified to classify more than 14 PQ events by appropriately setting the intermediate layers. 


\begin{tabular}{|c|c|c|c|c|c|c|c|}
\hline $\mathrm{Db}$ & $\begin{array}{c}80 \\
10.0 \% \\
\end{array}$ & $\begin{array}{c}0 \\
0.0 \%\end{array}$ & $\begin{array}{c}0 \\
0.0 \%\end{array}$ & $\begin{array}{c}0 \\
0.0 \%\end{array}$ & $\begin{array}{c}0 \\
0.0 \%\end{array}$ & $\begin{array}{c}0 \\
0.0 \%\end{array}$ & $\begin{array}{l}100 \% \\
0.0 \%\end{array}$ \\
\hline \multirow[t]{2}{*}{$\mathrm{Cc}$} & 0 & 80 & 0 & 2 & 0 & 0 & $97.5 \%$ \\
\hline & $0.0 \%$ & $10.0 \%$ & $0.0 \%$ & $0.2 \%$ & $0.0 \%$ & $0.0 \%$ & $2.5 \%$ \\
\hline \multirow[t]{2}{*}{$\mathrm{Da}$} & 0 & 0 & 80 & 0 & 2 & 0 & $97.5 \%$ \\
\hline & $0.0 \%$ & $0.0 \%$ & $10.0 \%$ & $0.0 \%$ & $0.2 \%$ & $0.0 \%$ & $2.5 \%$ \\
\hline \multirow[t]{2}{*}{$\mathrm{Cb}$} & 0 & 0 & 0 & 78 & 0 & 0 & $100 \%$ \\
\hline & $0.0 \%$ & $0.0 \%$ & $0.0 \%$ & $9.8 \%$ & $0.0 \%$ & $0.0 \%$ & $0.0 \%$ \\
\hline \multirow[t]{2}{*}{ D } & 0 & 0 & 0 & 0 & 78 & 0 & $100 \%$ \\
\hline & $0.0 \%$ & $0.0 \%$ & $0.0 \%$ & $0.0 \%$ & $9.8 \%$ & $0.0 \%$ & $0.0 \%$ \\
\hline \multirow[t]{5}{*}{$\mathrm{Ca}$} & 0 & 0 & 0 & 0 & 0 & 80 & $100 \%$ \\
\hline & $0.0 \%$ & $0.0 \%$ & $0.0 \%$ & $0.0 \%$ & $0.0 \%$ & $10.0 \%$ & $0.0 \%$ \\
\hline & $100 \%$ & $100 \%$ & $100 \%$ & $97.5 \%$ & $97.5 \%$ & $100 \%$ & $99.16 \%$ \\
\hline & $0.0 \%$ & $0.0 \%$ & $0.0 \%$ & $2.5 \%$ & $2.5 \%$ & $0.0 \%$ & $0.84 \%$ \\
\hline & $\mathrm{Db}$ & $\mathrm{Cc}$ & $\mathrm{Da}$ & $\mathrm{Cb}$ & $\mathrm{Dc}$ & $\mathrm{Ca}$ & \\
\hline
\end{tabular}

Figure 8. Confusion matrix of training and validation

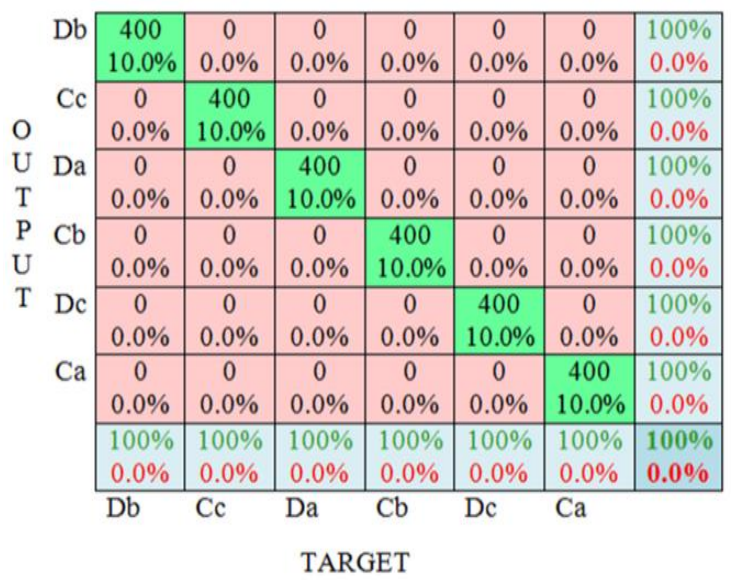

Figure 9. Confusion matrix for test data set 2

Table 5. Performance of CNN model - CR and FAR

\begin{tabular}{cccccccc}
\hline Class & $\mathrm{Db}$ & $\mathrm{Cc}$ & $\mathrm{Da}$ & $\mathrm{Cb}$ & $\mathrm{Dc}$ & $\mathrm{Ca}$ & Avg. \\
\hline CR\% & 98.9 & 99.5 & 98.5 & 92.3 & 96.8 & 97.4 & 97.23 \\
FAR $\%$ & 0.9 & 0.9 & 0.2 & 0.4 & 0.3 & 0.2 & 0.48 \\
\hline
\end{tabular}

Table 6. Comparison of classification models for PQ events

\begin{tabular}{ccccccc}
\hline Method & $\begin{array}{c}\text { Feature } \\
\text { extraction }\end{array}$ & $\begin{array}{c}\text { No. of PQ } \\
\text { events }\end{array}$ & $\begin{array}{c}\text { Accuracy } \\
(\%)\end{array}$ & $\begin{array}{c}\text { Training } \\
\text { Time }\end{array}$ & $\begin{array}{c}\text { Computation } \\
\text { time }\end{array}$ & References \\
\hline PSR+CNN & Automated & 10 & 99.8 & $113 \mathrm{~min}$ & $7 \mathrm{~min}$ & {$[18]$} \\
SPM+CNN & Automated & 7 & 96.78 & $98 \mathrm{~min}$ & $5 \mathrm{~min}$ & {$[20]$} \\
ST+NN+DT & Handcrafted & 13 & 99.9 & - & - & {$[24]$} \\
Hybrid ST + DT & Handcrafted & 11 & 94.9 & - & - & {$[25]$} \\
SPM+DTCWT+CNN & Automated & 6 & 97.23 & $13.12 \mathrm{~min}$ & $23 \mathrm{sec}$ & Proposed \\
\hline
\end{tabular}

\section{CONCLUSION}

A novel algorithm is presented in this paper that is used to classify voltage variations into 6 different PQ events considering the SPM diagrams, DTCWT sub bands and the CNN model. The input data which is of 1D is converted into 2D using SPM and the intensity components and the directional features are separated using DTCWT. The low pass and high pass sub bands are independently trained by the customized CNN model to perform classification of the PQ events. Compared with the reported PQ event classification methods the proposed algorithm is demonstrated to achieve higher accuracy and is fast in classification of events with reduced complexity in the architecture.

\section{REFERENCES}

[1] M. H. Rehmani, M. Reisslein, A. Rachedi, M. Erol-Kantarci, and M. Radenkovic, "Integrating renewable energy resources into the smart grid: Recent developments in information and communication technologies," IEEE Transactions on Industrial Informatics, vol. 14, no. 7, pp. 2814-2825, 2018, doi: 10.1109/TII.2018.2819169.

[2] M. H. Bollen et al., "Power quality concerns in implementing smart distribution-grid applications," IEEE Transactions on Smart Grid, vol. 8, no. 1, pp. 391-399, 2016, doi: 10.1109/TSG.2016.2596788.

[3] S. Mohibullah and S. Laskar, "Power quality issues and need of intelligent PQ monitoring in the smart grid environment," in 2012 47th International Universities Power Engineering Conference (UPEC) 2012, pp. 1-6, doi: 10.1109/UPEC.2012.6398437.

[4] A. R. A. Jerin, N. Prabaharan, N. M. Kumar, K. Palanisamy, S. Umashankar, and P. Siano, "Smart grid and power quality issues," in Hybrid-Renewable Energy Systems in Microgrids: Elsevier, pp. 195-202, 2018, doi: 10.1016/B978-0-08-102493-5.00010-8.

[5] A. E. Legarreta, J. H. Figueroa, and J. A. Bortolin, "An IEC 61000-4-30 class A-Power quality monitor: Development and performance analysis," in 11th International Conference on Electrical Power Quality and Utilisation, 2011, pp. 1-6, doi: 10.1109/EPQU.2011.6128813.

[6] C. Hemapriya, M. Suganyadevi, and C. Krishnakumar, "Detection and classification of multi-complex power quality events in a smart grid using Hilbert-Huang transform and support vector machine," Electrical Engineering, vol. 102, no. 3, pp. 1681-1706, 2020, doi: 10.1007/s00202-020-00987-8.

[7] S. D. Hole and C. A. Naik, "Power Quality Events' Classification employing Discrete Wavelet Transform and Machine Learning," in 2020 First IEEE International Conference on Measurement, Instrumentation, Control and Automation (ICMICA), 2020, pp. 1-5, doi: 10.1109/ICMICA48462.2020.9242894. 
[8] O. P. Mahela, A. G. Shaik, B. Khan, R. Mahla, and H. H. Alhelou, "Recognition of Complex Power Quality Disturbances Using S-Transform Based Ruled Decision Tree," IEEE Access, vol. 8, pp. 173530-173547, 2020, doi: 10.1109/ACCESS.2020.3025190.

[9] I. S. Samanta, P. K. Rout, and S. Mishra, "An optimal extreme learning-based classification method for power quality events using fractional Fourier transform," Neural Computing and Applications, vol. 33, no. 10, pp. 4979-4995, 2021, doi: 10.1007/s00521-020-05282-y.

[10] B. Choudhary, "An Advanced Genetic Algorithm with Improved Support Vector Machine for Multi-Class Classification of Real Power Quality Events," Electric Power Systems Research, vol. 191, 2021, Art. no. 106879, doi: 10.1016/j.epsr.2020.106879.

[11] H. Liu, F. Hussain, S. Yue, O. Yildirim, and S. J. Yawar, "Classification of multiple power quality events via compressed deep learning," International Transactions on Electrical Energy Systems, vol. 29, no. 6, 2019, Art. no. e12010, doi: 10.1002/20507038.12010

[12] K. R. Reddy, "Power Quality Classification of disturbances using Discrete Wavelet Packet Transform (DWPT) with Adaptive Neuro-Fuzzy System," Turkish Journal of Computer and Mathematics Education (TURCOMAT), vol. 12, no. 3, pp. 4892-4903, 2021, doi: 10.17762/turcomat.v12i3.1995.

[13] M. Guder, O. Salor, I. Çadirci, B. Ozkan, and E. Altintas, "Data mining framework for power quality event characterization of iron and steel plants," IEEE Transactions on Industry Applications, vol. 51, no. 4, pp. 3521-3531, 2015, doi: 10.1109/TIA.2015.2406751.

[14] A. El-Zonkoly, "Expert System for Classification of Power System Events.(Dept. E)," MEJ. Mansoura Engineering Journal, vol. 29, no. 3, pp. 43-50, 2021, doi: 10.21608/bfemu.2021.140367.

[15] E. Styvaktakis, M. H. Bollen, and I. Y.-H. Gu, "Expert system for voltage dip classification and analysis," in 2001 Power Engineering Society Summer Meeting. Conference Proceedings (Cat. No. 01CH37262), vol. 1, 2001, pp. 671-676, doi: 10.1109/PESS.2001.970122.

[16] H. Erişti and Y. Demir, "Automatic classification of power quality events and disturbances using wavelet transform and support vector machines," IET generation, transmission and distribution, vol. 6, no. 10, pp. 968-976, 2012.

[17] E. Balouji and O. Salor, "Classification of power quality events using deep learning on event images," in 20173 rd International Conference on Pattern Recognition and Image Analysis (IPRIA), 2017, pp. 216-221, doi: 10.1109/PRIA.2017.7983049.

[18] K. Cai, T. Hu, W. Cao, and G. Li, "Classifying power quality disturbances based on phase space reconstruction and a convolutional neural network," Applied Sciences, vol. 9, no. 18, 2019, Art. no. 3681, doi: 10.3390/app9183681.

[19] A. Bagheri, M. H. Bollen, and I. Y. Gu, "Improved characterization of multi-stage voltage dips based on the space phasor model," Electric Power Systems Research, vol. 154, pp. 319-328, 2018, doi: 10.1016/j.epsr.2017.09.004.

[20] A. Bagheri, I. Y. Gu, M. H. Bollen, and E. Balouji, "A robust transform-domain deep convolutional network for voltage dip classification," IEEE Transactions on Power Delivery, vol. 33, no. 6, pp. 2794-2802, 2018, doi: 10.1109/TPWRD.2018.2854677.

[21] I. W. Selesnick, R. G. Baraniuk, and N. C. Kingsbury, "The dual-tree complex wavelet transform," IEEE signal processing magazine, vol. 22, no. 6, pp. 123-151, 2005, doi: 10.1109/MSP.2005.1550194.

[22] N. L. Kurva and S. Varadarajan, "Dual tree complex wavelet transform based image denoising for Kalpana satellite images," International Journal of Engineering and Technology, vol. 7, no. 3.29, pp. 269-271, 2018.

[23] J. Sierra-Fernández, J. G. De La Rosa, A. Agüera-Pérez, J. P. Salas, and O. Florencias-Oliveros, "Evaluation of a new Power Quality index, based in Higher Order Statistics," Renewable Energy and Power Quality Journal, pp. 37-42, 2017.

[24] R. Kumar, B. Singh, D. Shahani, A. Chandra, and K. Al-Haddad, "Recognition of power-quality disturbances using S-transformbased ANN classifier and rule-based decision tree," IEEE Transactions on Industry Applications, vol. 51, no. 2, pp. 1249-1258, 2014, doi: 10.1109/TIA.2014.2356639.

[25] M. Biswal and P. K. Dash, "Detection and characterization of multiple power quality disturbances with a fast S-transform and decision tree based classifier," Digital Signal Processing, vol. 23, no. 4, pp. 1071-1083, 2013, doi: 10.1016/j.dsp.2013.02.012. 